\title{
Identify differential gene expressions in fatty infiltration process in rotator cuff
}

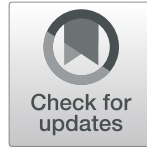

Pengfei $\mathrm{Hu}^{1,2} \mathbb{D}$, Lifeng Jiang ${ }^{1,2}$ and Lidong $\mathrm{Wu}^{1 *}$

\begin{abstract}
Background: Rotator cuff tears are one of the most frequent upper extremity injuries and lead to pain and disability. Recent studies have implicated fatty infiltration in rotator cuff is a key failure element with the higher retear rates and poorer functional prognosis. Therefore, we investigated the differential expression of key genes in each stage of rotator cuff tear.

Methods: A published expression profile was downloaded from the Gene Expression Omnibus database and analyzed using the Linear Models for Microarray Data (LIMMA) package in R language to identify differentially expressed genes (DEGs) in different stages of injured rotator cuff muscles. Gene ontology (GO) functional and Kyoto Encyclopedia of Genes and Genomes (KEGG) pathway analyses were performed to annotate the function of the DEGs. Finally, PPI network and module analysis were used to identify hub genes.

Results: A total of 1089 fatty infiltration-related DEGs were identified, including 733 upregulated and 356 downregulated genes, and GO analyses confirmed that fatty infiltration was strongly associated with inflammatory response, aging, response to lipopolysaccharide, and immune response. Significantly enriched KEGG pathways associated with these DEGs included the phagosome, cell adhesion molecules, tuberculosis, and osteoclast differentiation. Further analyses via a PPI network and module analysis identified a total of 259 hub genes. Among these, Tmprss11d, Ptprc, Itgam, Mmp9, TIr2, II1 b, II18, Ccl5, Cxcl10, and Ccr7 were the top ten hub genes.
\end{abstract}

Conclusions: Our findings indicated the potential key genes and pathways involved in fatty degeneration in the development of fatty infiltration and supplied underlying therapeutic targets in the future.

Keywords: Rotator cuff tears, Fatty infiltration, DEGs, GO annotation, KEGG pathway, Module analysis

\section{Background}

Rotator cuff tear (RCT) is one of the most common musculoskeletal disorder in orthopedics department. Earlier in 1991, the prevalence of shoulder disorders in people aged 70 and above was $21 \%$ [1]. According to a survey performed by Hiroshi in 2012, the prevalence of RCT was $22.1 \%$ was in the general population, and the prevalence was significantly increased with advanced age [2]. The disease is characterized as injury of one or more tendons/muscles in rotator cuff, which can cause pain, stiffness, muscle weakness, and functional limitation. Common risk factors for RCT include advancing age, overuse of shoulder, trauma, and shoulder arthritis [3].

\footnotetext{
* Correspondence: wulidong@zju.edu.cn

${ }^{1}$ Department of Orthopedic Surgery, Second Affiliated Hospital, School of Medicine, Zhejiang University, 88 Jiefang Road, Hangzhou, Zhejiang 310009, People's Republic of China

Full list of author information is available at the end of the article
}

Surgery is an effective solution for patients with large and full-thickness RCT. Currently, surgical procedures include mini-open and all-arthroscopic rotator cuff repair. According to the follow-up results, there is no significant difference in clinical prognosis between the two methods [4]. However, the failure rate was reported ranged from 23 to $77 \%$ in arthroscopically repaired massive rotator cuff tears, which characterized as the size of tear was more than $5 \mathrm{~cm}$ or the tear involving more than two cuff tendons [5-7]. The size of the defect, tendon retraction, muscular atrophy, and degree of fatty degeneration were regarded as failure elements and resulted in higher rate of no-heal and re-tear [8, 9]. Fatty infiltration was introduced and staged by Goutallier et al. in 1994 and was viewed as a key factor responsible for the higher re-tear rates and poorer functional prognosis $[10,11]$. Fatty degeneration of the infraspinatus

(c) The Author(s). 2019 Open Access This article is distributed under the terms of the Creative Commons Attribution 4.0 International License (http://creativecommons.org/licenses/by/4.0/), which permits unrestricted use, distribution, and 
was reported as the most independent predictor on outcomes following rotator cuff repair [12]. Chung et al. also found fatty infiltration of the infraspinatus is a significantly positive correlated factor with healing failure rate after arthroscopic repair of a massive rotator cuff tear [6]. Another cohort study performed by Gladstone et al. demonstrated that both muscle atrophy and fatty infiltration of the rotator cuff muscles cast significant impacts on regulating cuff repair and functional recovery [13]. Therefore, an increasing large number of research groups are focusing on the specific mechanism of fatty infiltration.

Till now, the key genes and pathway involved in the fatty infiltration of rotator cuff tear still unclear. Ren et al. identified differentially expressed genes and signaling pathways involved in rotator cuff tear through bioinformatics analysis [14]. Their study focused on the different genes between torn tendon samples and intact samples. In the present study, to explore differential gene expression of the differential stage of RCT, we examined one published gene dataset available in the Gene Expression Omnibus (GEO) database. Differential expression of genes during each developmental stages of massive rotator cuff tear was examined by comprehensive bioinformatics analyses. Then Gene Ontology (GO) and Kyoto Encyclopedia of Genes and Genomes (KEGG) pathway enrichment analyses were performed, and a protein-protein interaction (PPI) network was used to screen for crucial genes and pathways in the pathophysiological processes of fatty infiltration. Chief sub-PPI networks are explored by MCODE.

\section{Methods}

\section{Expression profile dataset}

Gene expression profiles for rats with a massive tear of the rotator cuff at different development stages (GSE103266) was obtained from the GEO database. In this experiment, rats were established by a bilateral full-thickness supraspinatus tear and suprascapular neurectomy and divided into four groups according to the period after operation: 0 days (un-operated controls), 10 days, 30 days, and 60 days post-injury. The count data in GSE103266 was normalized by DESeq2 package in R software and presented in $\log _{2}$ (TPM) [15]. The quality of gene expression data was analyzed and visualized using the ggplot2 package of $\mathrm{R}$ software for each sample. This research has been approved by the IRB of the authors' affiliated institutions.

\section{Differential gene expression analyses}

We compared the different three stages of rats (10 days, 30 days, and 60 days after operation) with un-operated controls to identify the DEGs. The Linear Models for Microarray Data (LIMMA) (limma) package, which includes
lmFit, eBayes, and topTable functions, was used for pairwise comparison of DEGs [16]. $P<0.05$ and abs $\left(\log _{2}\right)$ fold change $(\mathrm{FC})>1$ were used as the cutoff criteria.

\section{GO annotation and KEGG pathway enrichment analyses of DEGs}

GO annotation is a classic method used in bioinformatics analyses to describe the normal, in vivo biological role of genes or gene products [17]. KEGG, a collection of databases representing our knowledge on the molecular interaction, reaction, and relation networks, was used to clarify the potential role of the DEGs [18]. GO functional analyses encompassing biological processes (BP), cell components (CC), and molecular functions (MF) was performed using the Database for Annotation, Visualization and Integrated Discovery (DAVID ver. 6.8), with $P<0.05$ used as a cutoff.

\section{PPI network construction and hub gene identification}

A PPI network for the DEGs identified above was built based on The Search Tool for the Retrieval of Interacting Genes (STRING, http://string-db.org) and Cytoscape (ver. 3.5.1) [19]. In the PPI network, genes served as "nodes" and the line segment between two nodes represented associated interactions. The color of the lines between genes indicates the degree of interaction. The Centiscape plugin was used to determine the degree of connectivity for each node in the PPI network. Genes with a degree $>10$ were defined as hub genes. Furthermore, module analysis was performed by using the MCODE plugin (version 1.3) to explore the most important clustering modules in the huge PPI network (degree cutoff $=5$, $\mathrm{k}$-core $=2$, node score cutoff $=0.2$, and max. Depth rom Seed: 100).

\section{Results \\ Data distribution analyses and DEG screening}

In GSE103266, a total of 17147 genes were detected in 16 samples. The expression values (ranging from 0 to 20 $\left.\log _{2}(\mathrm{TPM})\right)$ and the distributions were similar between the four groups (Fig. 1a, b). H-cluster analyses and principal component showed that samples were easily grouped into different groups (Fig. 1c, d). Confirmed by these data distribution analyses, further bioinformatics analyses could be performed based on the available data.

Following data processing using limma, we identified 2877 DEGs (1554 upregulated, 1323 downregulated) in group 0 day vs. group 10 days (Fig. 2b), 2743 DEGs (1711 upregulated, 1032 downregulated) in group 0 day vs. group 30 days (Fig. 2c), and 2199 DEGs (1439 upregulated, 760 downregulated) in group 0 day vs. group 60 days (Fig. 2d). 

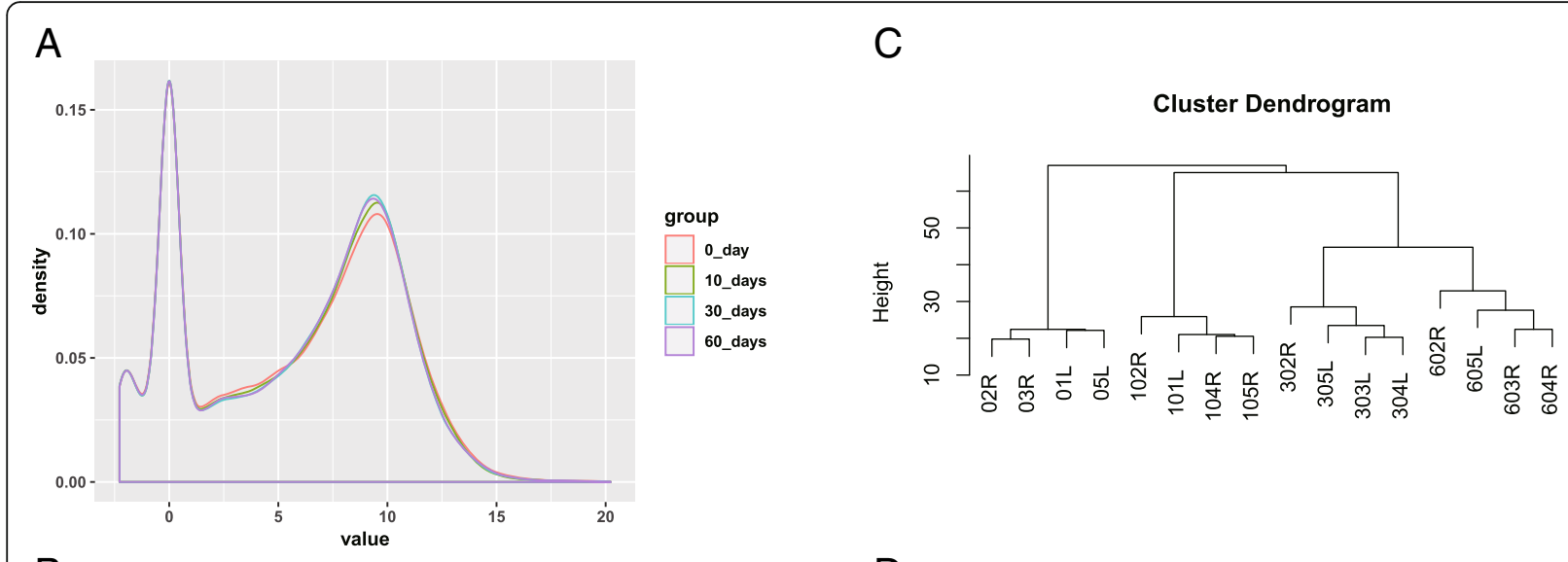

B
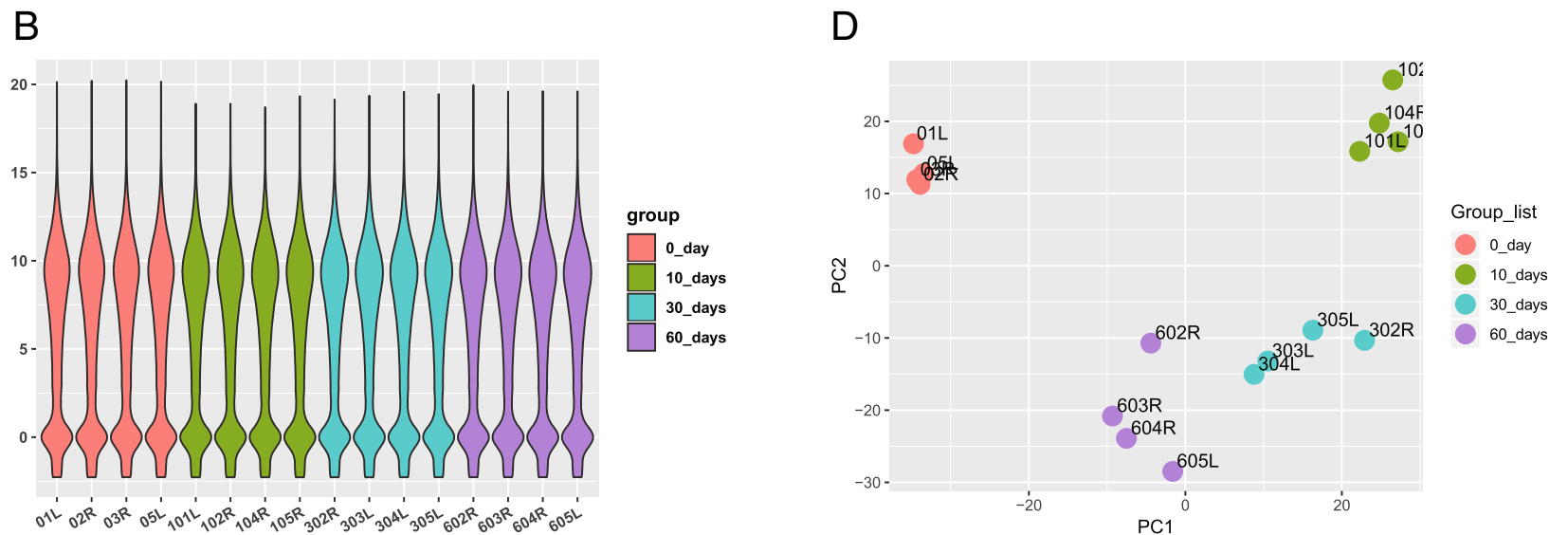

Fig. 1 Distribution analyses of gene expression. a Distribution of gene expression levels in GSE103266. b Distribution of genes expression of 16 samples. c Cluster analyses for all samples. d Principle component analyses of each samples

\section{Intersection between the DEGs}

We identified a total of 1089 DEGs common across each of the different stages by intersecting the three comparisons above (Fig. 3a), including 733 upregulated and 356 downregulated genes (Additional file 1: Table S1). Hierarchical clustering of the identified DEGs is displayed as a heatmap in Fig. 3b.

\section{GO annotation and KEGG pathway enrichment analyses}

GO annotation and KEGG pathway enrichment analyses were performed to better understand the functional significance of the DEGs. DEGs mainly partcipated in biological processes (BP) regarding response to drug, inflammatory response, aging, response to lipopolysaccharide and immune response. Significant cellular component (CC) terms revealed DEGs were principally enriched in extracellular exosome, extracellular space, and cell surface. Finally, the top five molecular functions (MF) identified were protein homodimerization activity, calcium ion binding, receptor binding, heparin binding, and carbohydrate binding (Fig. 4a). All significant biological processes-enriched entries are shown in a histogram in Fig. 4b. KEGG analyses of the DEGs revealed mainly enrichment for ten terms
(Fig. 5a). The top five significantly enriched KEGG pathways were the HTLV-I infection, phagosome, cell adhesion molecules, tuberculosis, and osteoclast differentiation. Detailed information regarding each of the DEGs involved in the KEGG pathway are listed in Fig. 5b.

\section{PPI network construction and module analysis}

We constructed a putative PPI network map for the overlapping DEGs using the STRING database and Cytoscape(Fig. 6). After degree calculation, a total of 259 hub genes were identified with the degree more than 10 , as listed in Additional file 2: Table S2. Among these, Tmprss11d, Ptprc, Itgam, Mmp9, Tlr2, Il1b, Il18, Ccl5, Cxcl10, and Ccr7 were the top ten hub genes according to the MCODE degree score.

A total of six modules were separated from the PPI network with both MCODE score $\geq 5$ and nodes $\geq 5$ (Fig. 7). KEGG analysis showed that the main pathways of these modules were associated with cytokine-cytokine receptor interaction, rheumatoid arthritis and toll-like receptor signaling pathway, osteoclast differentiation, metabolic pathways, fatty acid degradation, and so on. 

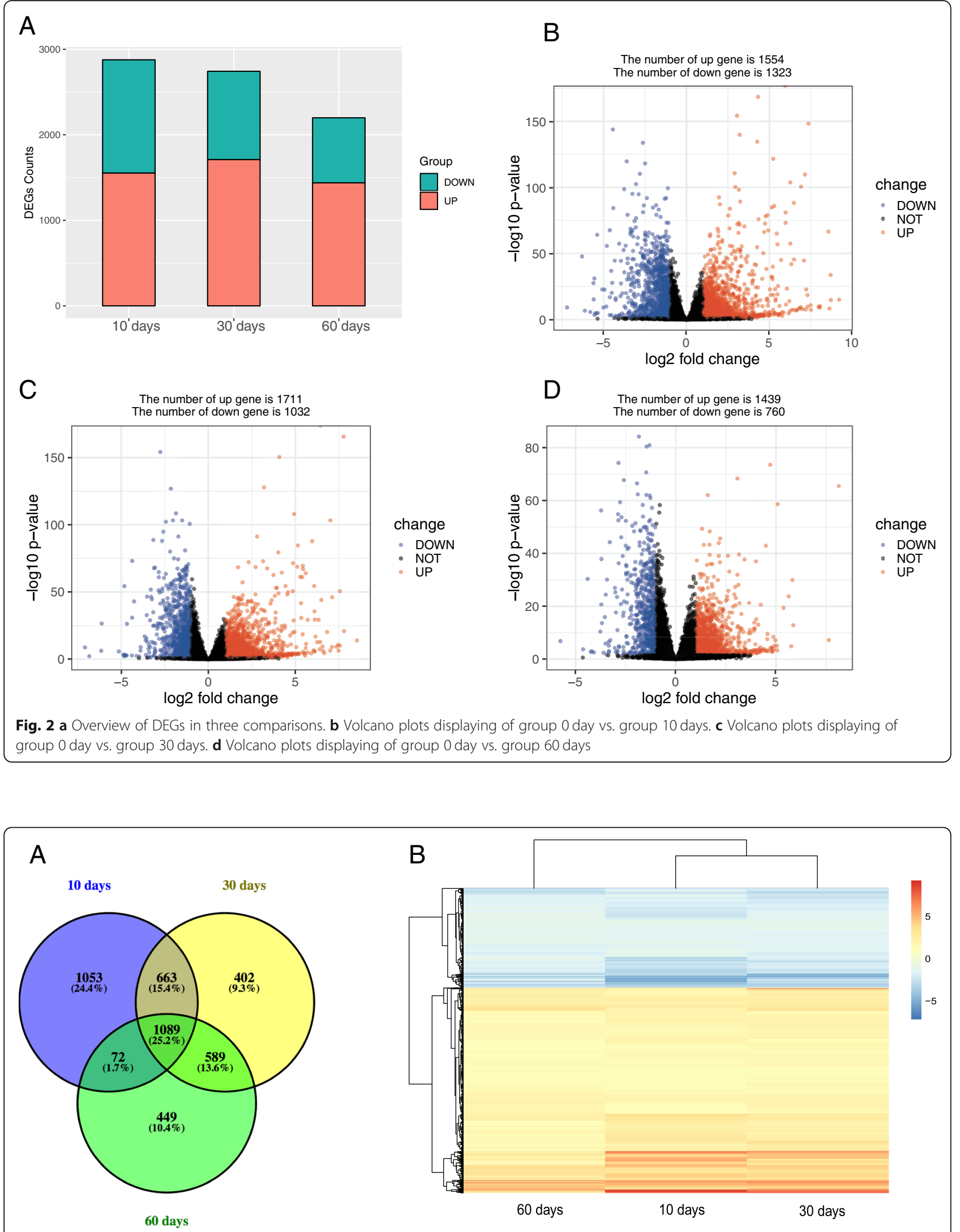

Fig. 3 a Venn diagram of DEGs across different stages. $\mathbf{b}$ Hierarchical clustering analyses of DEGs common to all groups 


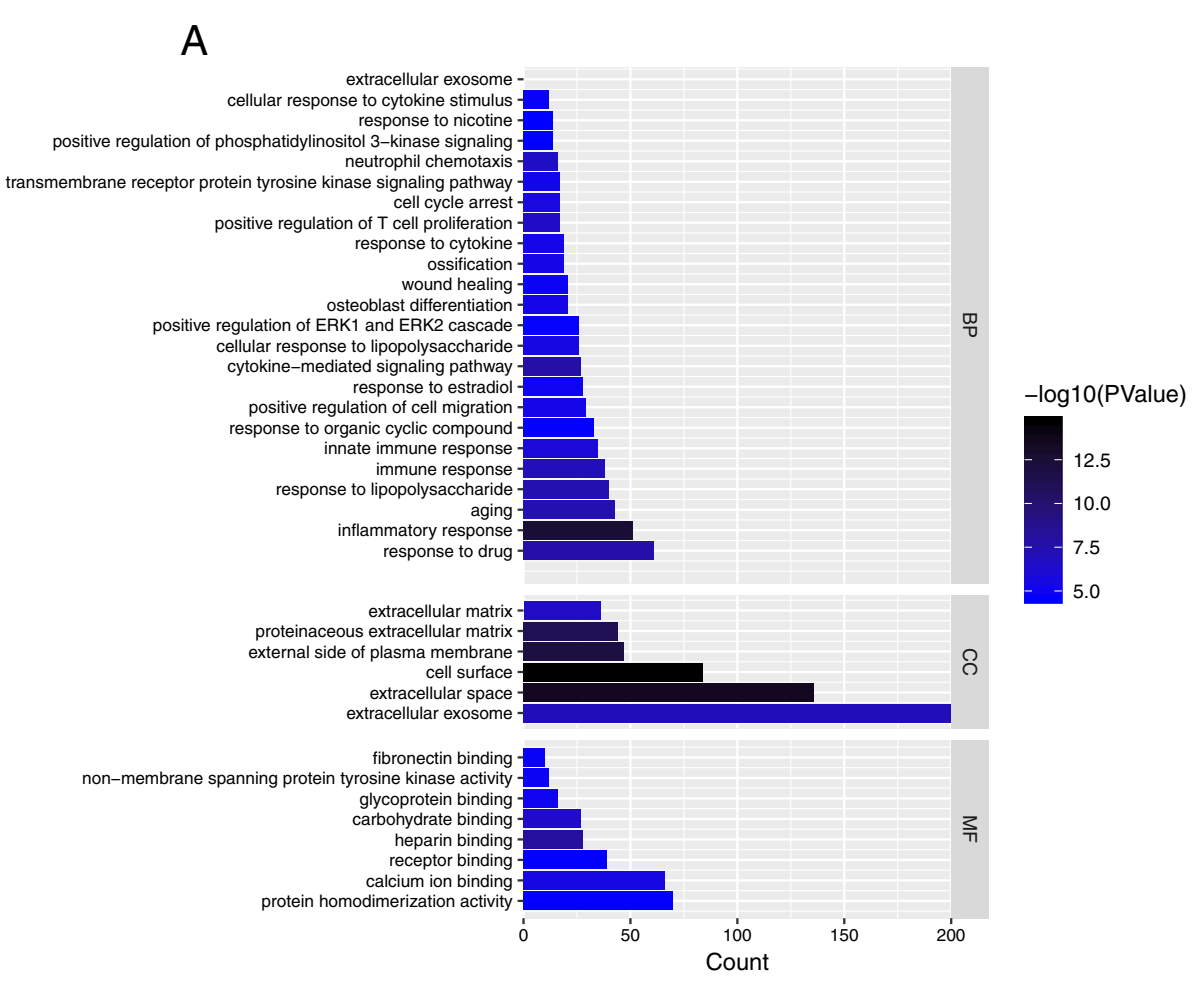

B

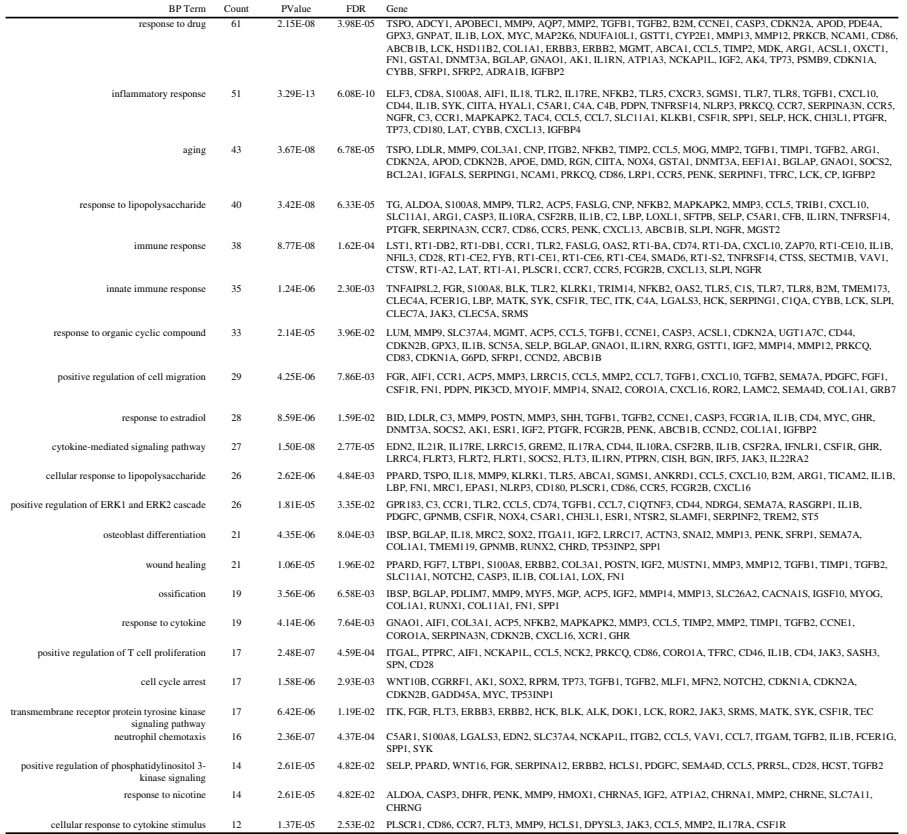

Fig. 4 a Histogram of Gene Ontology (GO) functional classification of DEGs. GO terms including biological processes (BP), cellular components (CC), and molecular functions (MF). b Top BP terms and their corresponding genes in GO functional enrichment analyses

\section{Discussion}

The mechanism of fatty infiltration of the rotator cuff is complex and still unclear. Using a mouse model constructed by unilateral supraspinatus and infraspinatus tendon tear, Wang et al. demonstrated atrophy and fatty infiltration of muscle exist in the 6-week delayed repair group. And the severity of fatty infiltration was closely linked with the shoulder function [20]. Another massive rotator cuff tear model caused by a full-thickness tenectomy of the right supraspinatus and infraspinatus in rats showed that the muscle fiber force is decreased and expressions of fibrosis and lipid accumulation related 


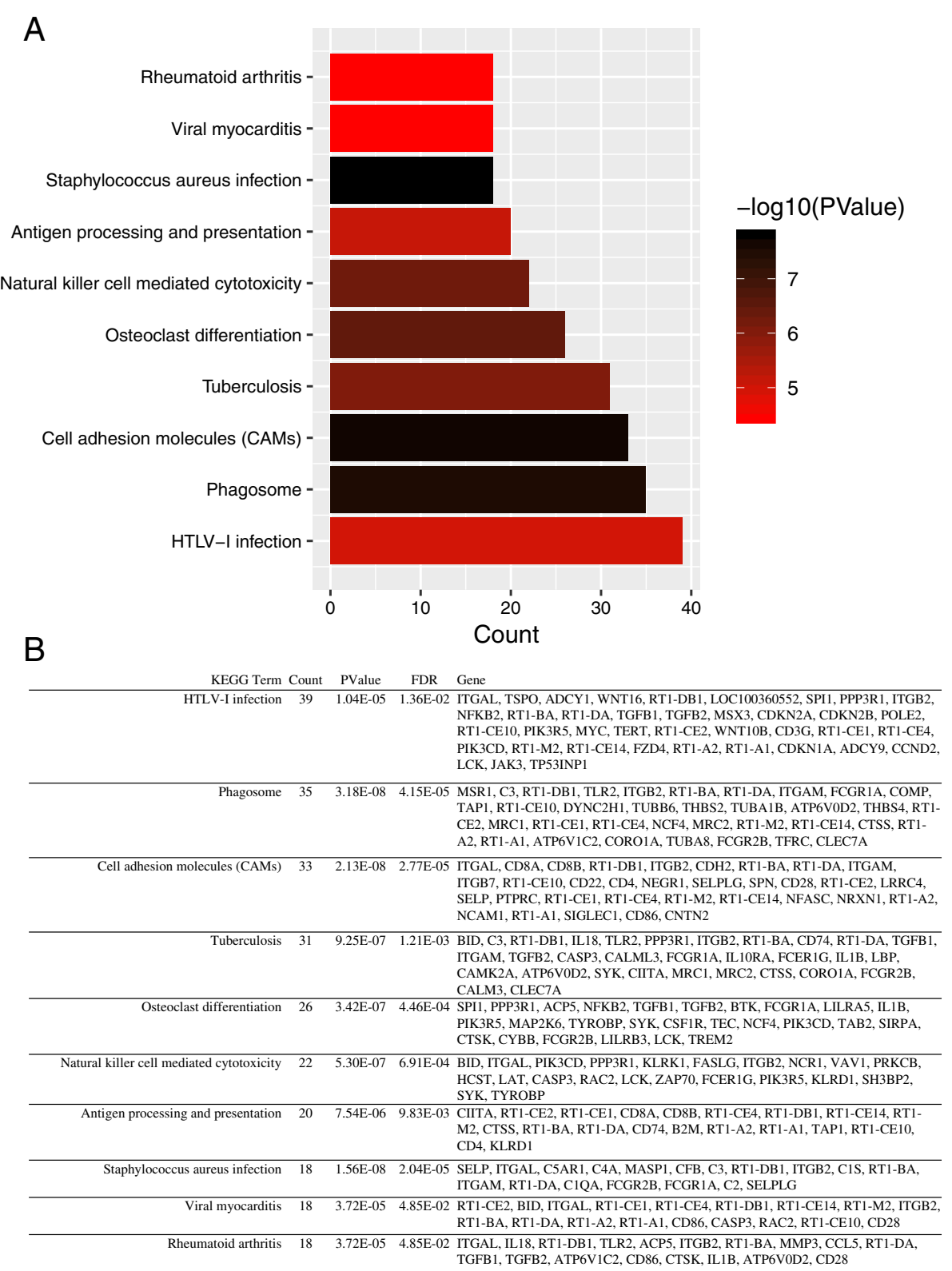

Fig. 5 a Histogram of KEGG pathways enrichment in DEGs. The graph displays only significantly enriched KEGG terms $(P<0.05)$, with darker red indicating greater significance. $\mathbf{b}$ Individual KEGG terms and their corresponding genes are shown for each group

genes were increased [21]. A further study performed by Liu et al. demonstrated that the main cellular sources involved in fatty infiltration were Tie 2 + progenitor cells and fibro/adipogenic progenitor cells [22]. These three studies were consistent with the clinical reports and researches. In a prospective study aimed to massive rotator cuff tears patients younger than 65 years, $67.9 \%$ were demonstrated with a stage 3 or 4 fatty infiltration of the supraspinatus muscle and $47.3 \%$ were suffered from a stage 3 or 4 fatty infiltration of the infraspinatus muscle [23]. And patients with a high degree of fatty infiltration exhibited poorer prognosis after surgery. Patients with grade 3 fatty degeneration showed significant improvement after arthroscopic rotator cuff repair compared to those patients who graded as Goutallier stage IV after nearly 3 years of follow-up [24]. However, a successful repair of rotator cuff would not bring a significant improvement of fatty infiltration and atrophy of the rotator cuff [13]. So, studies focused on the cellular and molecular basis of fatty infiltration are of great significance in the prevention and treatment of fatty infiltration in rotator cuff tear.

In the present study, a bioinformatics analysis was used to analyze an available database downloaded from 


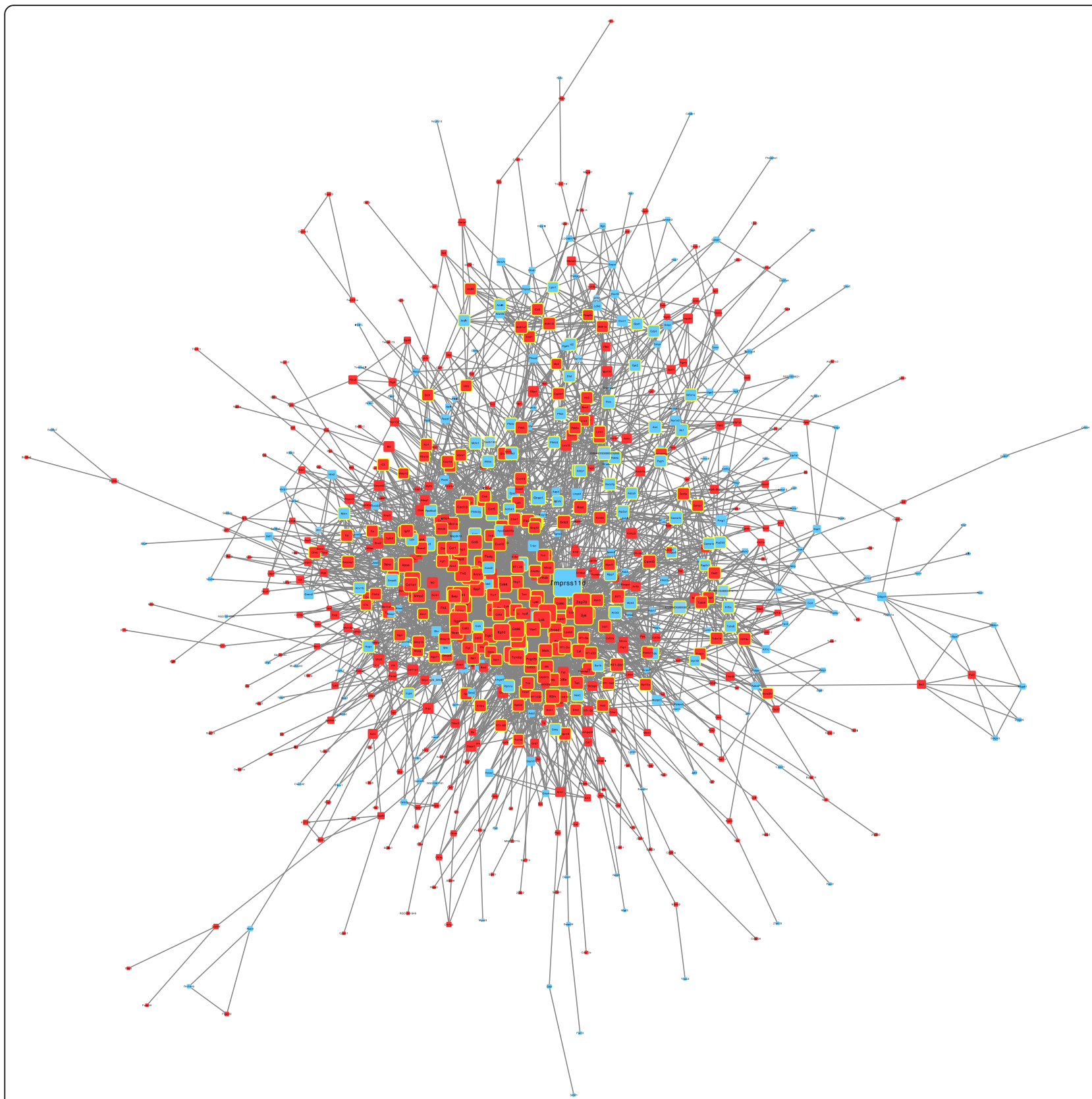

Fig. 6 Protein-protein interaction (PPI) network construction. Genes with yellow circles represent hub genes with a degree of interaction $\geq 10$. A total of 259 DEGs were identified as fatty infiltration-related hub genes

GEO. According to our results, a total of 1089 DEGs were identified as the key mediators in the pathophysiological processes of fatty infiltration. Moreover, we also performed GO annotation and KEGG pathway enrichment analyses to potential biological functions and pathways involved in fatty infiltration. In addition, 259 hub genes including Tmprss11d, Ptprc, Itgam, Mmp9, Tlr2, Il1b, Il18, Ccl5, Cxcl10, and Ccr7 and six modules with important functions were identified from the PPI network.
At present, there are only a few theories and research results about the mechanism of fatty infiltration in rotator cuff. Tmprss11d, a member of type II transmembrane serine protease (TTSP) family, was highly expressed in respiratory epithelium and proved to exert an important role in the host defense system [25]. Till now, the role of TMPRSS11D in the musculoskeletal system was not clear. Cao et al. reported higher expression of TMPRSS11D is closely linked with the severity of the cancer because of its ability to disintegrate the 


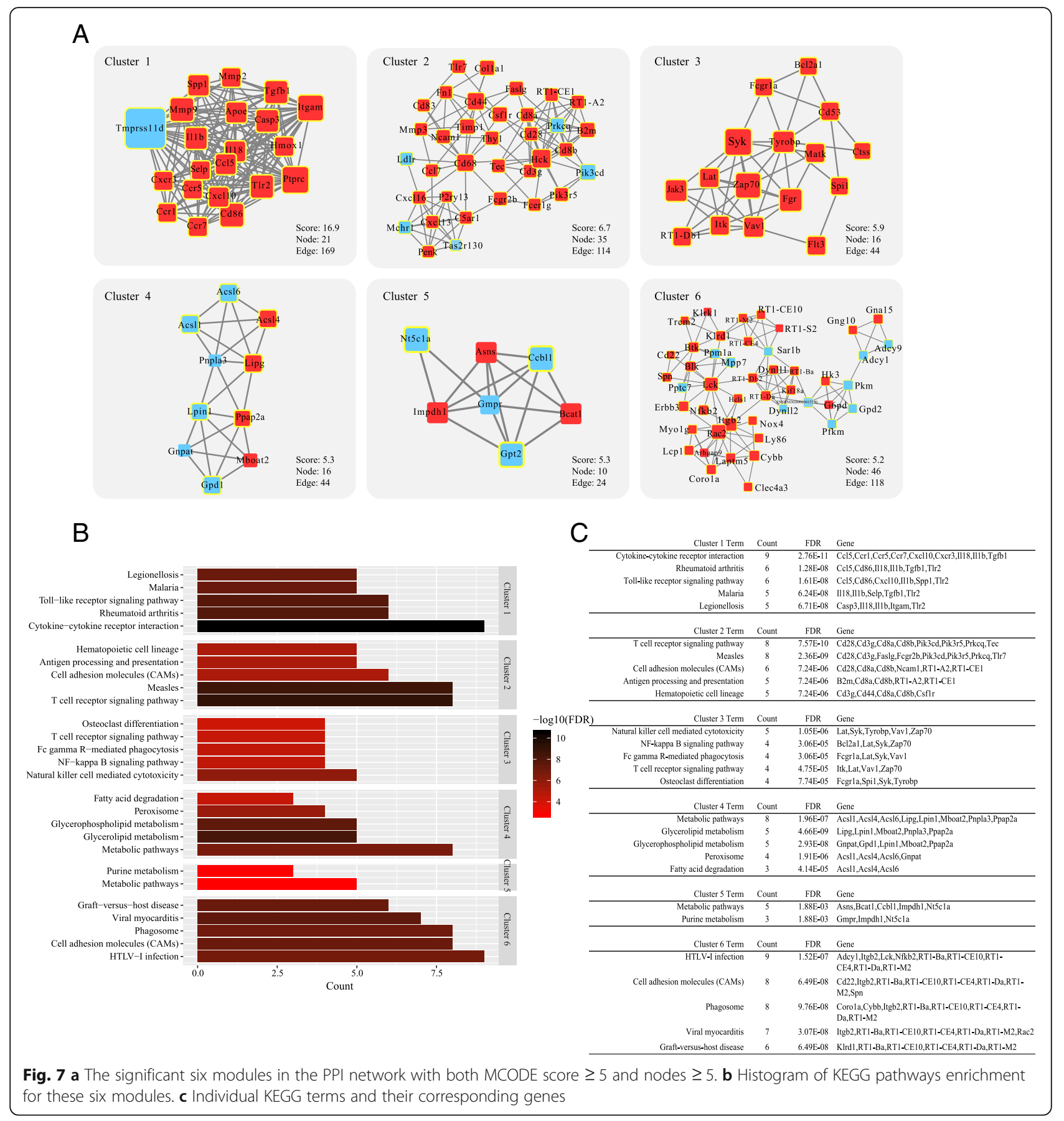

extracellular matrix [26]. TMPRSS11D was also demonstrated to have a relationship with fat metabolism in the liver [25]. Therefore, it is an important research direction of the role of TMPRSS11D in the degeneration of tendon and lipid metabolism in the future. MMP family was also identified as a major player in rotator cuff tear. Protein expressions of MMP-9 and MMP-13 were notably increased in rotator cuff specimens obtained from surgery patients [27]. However, in MMP-13 (-/-) knockout mice group, researchers found a higher amount of fat infiltration compared to the wildtype MMP-13 $(+/+)$ mice group. These interesting results indicated that MMP-13 exert a significant role in rotator muscle fatty infiltration and may be a potential target for treatment [28]. MMP inhibitor was helpful in the improvement of tendon-to-bone healing in a rotator cuff repair model [29]. Moreover, Tlr2, another identified hub gene in our study, was highly expressed in rotator cuff tendon in the first 2 weeks following injuries [30]. Tlr2 was viewed as an important mediator in NLRP3 inflammasome. The 
lowered activity of NLRP3 leads to a decrease in the fat accumulation in hepatocytes [31]. So, the molecular mediators associated with NLRP3 inflammasome was worth studying in the pathology of fatty infiltration in rotator cuff tendons. Taken together, hub genes identified in our research by comprehensive bioinformatic analyses were meaningful and worthy of deep-going study in the future.

We also performed an intensive module analysis based on the PPI network in this study. In chronically rotator cuff muscles, increased gene expressions of PPAR- $\gamma$, PLD1, miR-27a and lipid synthesis were obversed. And ECM degradation- and remodeling-related genes such as MMP2, MMP9, and MMP14 were also overexpressed in the pathological process of fibrosis [32]. Blocking of p38 MAPK signaling, an important regulator of adipogenesis, reduced the fat accumulation by nearly half and adjusted the expressions of adipogenesis- and matrix accumulation-related genes [33]. Our KEGG pathway enrichment analyses showed that the functions of module 4 and 5 were mainly associated with metabolic pathways and fatty acid degradation. And DEGs such as Acs11, Acs14, Acsl6, Lipg, Lpin1, Mboat2, Pnpla3, Ppap2a, Ccbl1, Impdh1, and Nt5c1a were functionality associated with these pathways.

A limitation of this study is that the stage of fatty infiltration in the rat models in GSE103266 is unclear. Besides, there are some anatomical differences between human and rat. Therefore, the pathophysiological process of fatty infiltration in rat is different from human. We still need to conduct validation experiment to proof our speculation in the future.

\section{Conclusion}

The current study identified a series of DEGs in each stage of massive rotator cuff tear. Using a series of bioinformatics analyses, hub genes including Tmprss11d, Ptprc, Itgam, Mmp9, Tlr2, Il1b, Il18, Ccl5, Cxcl10, and Ccr7 were identified. Further GO and KEGG pathway analysis suggests potential mechanisms through which these hub genes mediate fatty infiltration pathogenesis in rotator cuff tear. In addition, module analysis highlights the important roles of metabolic pathways and fatty acid degradation in the regulation of fatty infiltration. Our results indicated the potential key genes and pathways involved in fatty degeneration and supplied therapeutic targets to improve clinical outcomes for patients who suffer from chronic rotator cuff tears and fatty infiltration.

\section{Additional files}

Additional file 1: Table S1. 1089 DEGs in each of the different stages of rotator cuff tear. (DOCX $148 \mathrm{~kb})$
Additional file 2: Table S2. Hub-genes identified by CentiScape (degree 25) among 1089 DEGs. (DOCX 35 kb)

\section{Abbreviations}

BP: Biological processes; CC: Cell components; DEGs: Differentially expressed genes; GEO: Gene Expression Omnibus; GO: Gene ontology; KEGG: Kyoto Encyclopedia of Genes and Genomes; LIMMA: Linear Models for Microarray data; MF: Molecular functions; PPI: Protein-protein interaction; RCT: Rotator cuff tear

\section{Acknowledgements}

Not applicable.

Funding

None

Availability of data and materials

The data was freely downloaded from the public GEO database.

\section{Authors' contributions}

WLD conceived the design of the study. HPF and JLF participated in the design of this study and performed the statistical analysis. They both contributed to the collection of background information. HPF drafted the manuscript. All authors read and approved the final manuscript.

Ethics approval and consent to participate

Not applicable. This paper does not involve research on humans.

Consent for publication

All authors read and approved the final manuscript and consented to publication.

\section{Competing interests}

The authors declare that they have no competing interest.

\section{Publisher's Note}

Springer Nature remains neutral with regard to jurisdictional claims in published maps and institutional affiliations.

\section{Author details}

${ }^{1}$ Department of Orthopedic Surgery, Second Affiliated Hospital, School of Medicine, Zhejiang University, 88 Jiefang Road, Hangzhou, Zhejiang 310009, People's Republic of China. ${ }^{2}$ Orthopedics Research Institute of Zhejiang University, Hangzhou, People's Republic of China.

Received: 12 March 2019 Accepted: 30 April 2019

Published online: 28 May 2019

\section{References}

1. Chard MD, Hazleman R, Hazleman BL, King RH, Reiss BB. Shoulder disorders in the elderly: a community survey. Arthritis Rheum. 1991;34:766-9.

2. Minagawa H, Yamamoto N, Abe H, Fukuda M, Seki N, Kikuchi K, et al. Prevalence of symptomatic and asymptomatic rotator cuff tears in the general population: from mass-screening in one village. J Orthop. 2013;10: 8-12.

3. Sayampanathan AA, Andrew TH. Systematic review on risk factors of rotator cuff tears. J Orthop Surg (Hong Kong). 2017;25:2309499016684318.

4. Oliva F, Piccirilli E, Bossa M, Via AG, Colombo A, Chillemi C, et al. I.S.Mu.L.T Rotator cuff tears guidelines. Muscles Ligaments Tendons J. 2015;5:227-63.

5. Lo IK, Burkhart SS. Arthroscopic revision of failed rotator cuff repairs: technique and results. Arthroscopy. 2004;20:250-67.

6. Chung SW, Kim JY, Kim MH, Kim SH, Oh JH. Arthroscopic repair of massive rotator cuff tears: outcome and analysis of factors associated with healing failure or poor postoperative function. Am J Sports Med. 2013;41:1674-83.

7. Gerber C, Fuchs B, Hodler J. The results of repair of massive tears of the rotator cuff. J Bone Joint Surg Am. 2000;82:505-15.

8. Rashid MS, Cooper C, Cook J, Cooper D, Dakin SG, Snelling S, et al. Increasing age and tear size reduce rotator cuff repair healing rate at 1 year. Acta Orthop. 2017;88:606-11. 
9. Valencia AP, Lai JK, lyer SR, Mistretta KL, Spangenburg EE, Davis DL, et al. Fatty infiltration is a prognostic marker of muscle function after rotator cuff tear. Am J Sports Med. 2018;46:2161-9.

10. Goutallier D, Postel JM, Bernageau J, Lavau L, Voisin MC. Fatty muscle degeneration in cuff ruptures. Pre- and postoperative evaluation by CT scan. Clin Orthop Relat Res. 1994;304:78-83.

11. Montgomery SR, Petrigliano FA, Gamradt SC. Failed rotator cuff surgery, evaluation and decision making. Clin Sports Med. 2012;31:693-712.

12. Oh JH, Kim SH, Ji HM, Jo KH, Bin SW, Gong HS. Prognostic factors affecting anatomic outcome of rotator cuff repair and correlation with functional outcome. Arthroscopy. 2009;25:30-9.

13. Gladstone JN, Bishop JY, Lo IK, Flatow EL. Fatty infiltration and atrophy of the rotator cuff do not improve after rotator cuff repair and correlate with poor functional outcome. Am J Sports Med. 2007;35:719-28.

14. Ren YM, Duan YH, Sun YB, Yang T, Tian MQ. Bioinformatics analysis of differentially expressed genes in rotator cuff tear patients using microarray data. J Orthop Surg Res. 2018;13:284.

15. Love Ml, Huber W, Anders S. Moderated estimation of fold change and dispersion for RNA-seq data with DESeq2. Genome Biol. 2014;15:550.

16. Ritchie ME, Phipson B, Wu D, Hu Y, Law CW, Shi W, et al. limma powers differential expression analyses for RNA-sequencing and microarray studies. Nucleic Acids Res. 2015;43:e47.

17. Ashburner M, Ball CA, Blake JA, Botstein D, Butler H, Cherry JM, et al. Gene ontology: tool for the unification of biology. The Gene Ontology Consortium. Nat Genet. 2000;25:25-9.

18. Kanehisa M, Goto S. KEGG: kyoto encyclopedia of genes and genomes. Nucleic Acids Res. 2000;28:27-30

19. Szklarczyk D, Franceschini A, Wyder S, Forslund K, Heller D, Huerta-Cepas J, et al. STRING v10: protein-protein interaction networks, integrated over the tree of life. Nucleic Acids Res. 2015:43:D447-52.

20. Wang Z, Liu X, Davies MR, Horne D, Kim H, Feeley BT. A mouse model of delayed rotator cuff repair results in persistent muscle atrophy and fatty infiltration. Am J Sports Med. 2018;46:2981-9.

21. Gumucio JP, Korn MA, Saripalli AL, Flood MD, Phan AC, Roche SM, et al. Aging-associated exacerbation in fatty degeneration and infiltration after rotator cuff tear. J Shoulder Elbow Surg. 2014;23:99-108.

22. Liu X, Ning AY, Chang NC, Kim H, Nissenson R, Wang L, et al. Investigating the cellular origin of rotator cuff muscle fatty infiltration and fibrosis after injury. Muscles Ligaments Tendons J. 2016;6:6-15.

23. Berhouet J, Collin P, Benkalfate T, Le Du C, Duparc F, Courage O, et al. Massive rotator cuff tears in patients younger than 65 years. Epidemiology and characteristics. Orthop Traumatol Surg Res. 2009:95:S13-8.

24. Burkhart SS, Barth JR, Richards DP, Zlatkin MB, Larsen M. Arthroscopic repair of massive rotator cuff tears with stage 3 and 4 fatty degeneration. Arthroscopy. 2007;23:347-54

25. Sales KU, Hobson JP, Wagenaar-Miller R, Szabo R, Rasmussen AL, Bey A, et al. Expression and genetic loss of function analysis of the HAT/DESC cluster proteases TMPRSS11A and HAT. PLoS One. 2011;6:e23261.

26. Cao X, Tang Z, Huang F, Jin Q, Zhou X, Shi J. High TMPRSS11D protein expression predicts poor overall survival in non-small cell lung cancer. Oncotarget. 2017;8:12812-9.

27. Jacob J, Eisemon E, Sheibani-Rad S, Patel A, Jacob T, Choueka J. Matrix metalloproteinase levels as a marker for rotator cuff tears. Orthopedics. 2012;35:e474-8

28. Liu X, Ravishankar B, Ning A, Liu M, Kim HT, Feeley BT. Knocking-out matrix metalloproteinase-13 exacerbates rotator cuff muscle fatty infiltration. Muscles Ligaments Tendons J. 2017;7:202-7.

29. Bedi A, Kovacevic D, Hettrich C, Gulotta LV, Ehteshami JR, Warren RF, et al. The effect of matrix metalloproteinase inhibition on tendon-to-bone healing in a rotator cuff repair model. J Shoulder Elbow Surg. 2010;19:384-91.

30. Thankam FG, Roesch ZK, Dilisio MF, Radwan MM, Kovilam A, Gross RM, et al. Association of inflammatory responses and ECM disorganization with HMGB1 upregulation and NLRP3 inflammasome activation in the injured rotator cuff tendon. Sci Rep. 2018;8:8918.

31. Yang $G$, Lee HE, Lee JY. A pharmacological inhibitor of NLRP3 inflammasome prevents non-alcoholic fatty liver disease in a mouse model induced by high fat diet. Sci Rep. 2016;6:24399.

32. Gumucio J, Flood M, Harning J, Phan A, Roche S, Lynch E, et al. T lymphocytes are not required for the development of fatty degeneration after rotator cuff tear. Bone Joint Res. 2014:3:262-72.
33. Wilde JM, Gumucio JP, Grekin JA, Sarver DC, Noah AC, Ruehlmann DG, et al. Inhibition of p38 mitogen-activated protein kinase signaling reduces fibrosis and lipid accumulation after rotator cuff repair. J Shoulder Elbow Surg. 2016:25:1501-8
Ready to submit your research? Choose BMC and benefit from:

- fast, convenient online submission

- thorough peer review by experienced researchers in your field

- rapid publication on acceptance

- support for research data, including large and complex data types

- gold Open Access which fosters wider collaboration and increased citations

- maximum visibility for your research: over $100 \mathrm{M}$ website views per year

At BMC, research is always in progress.

Learn more biomedcentral.com/submissions 